\title{
An improved automated method for the measurement of thermal thresholds. 2. patients with peripheral neuropathy
}

\author{
GORAN A JAMAL, ANDREW I WEIR, STIG HANSEN, JOHN P BALLANTYNE
}

From the Glasgow University Department of Neurology Institute of Neurological Sciences Southern General Hospital, and West Scotland Health Board's Department of Clinical Physics and Bio-engineering Glasgow, Scotland, $U K$

SUMMARY Thermal thresholds were determined by a new technique, at wrists and ankles in 143 patients with peripheral neuropathies of diverse aetiologies. Ninety-nine percent of patients (141/143) had abnormalities of one or both thresholds. In only two patients with mild/early Friedreich's ataxia were thermal thresholds normal. Electromyography was performed and fastest motor nerve conduction velocities and sensory nerve action potential parameters were measured in all the patients using conventional techniques in ulnar, median and sural nerves. Eighty-nine percent of patients (127/143) had one or more abnormalities on these electrophysiological studies. However, 39 of 40 patients with completely normal sensory nerve studies had an abnormality of one or more thermal thresholds. Eighty-six percent of 48 patients with normal sural nerve studies had abnormal thermal thresholds at the ankle. Sixty percent of 70 patients with normal sensory median and ulnar nerve studies had abnormal wrist thermal thresholds. This improved technique for the determination of thermal thresholds reveals that disturbances of thermal sensibility are present in the majority of peripheral neuropathies irrespective of aetiology. In some patients disturbances of thermal thresholds antedate the appearance of abnormalities on conventional electrophysiological investigation. The findings suggest that this technique has considerable usefulness in the detection of small nerve fibre dysfunction in the context of generalised neuropathy.

Thermal sensation depends on impulses reaching the central nervous system by adequate stimulation of thermal receptors. These impulses are carried exclusively in the non-myelinated $(\mathrm{C})$ and the thinly myelinated $(\mathrm{A} \delta)$ fibres. ${ }^{\prime}$ Activity in these fibres is not adequately tested by conventional electrophysiological methods.

The literature on the measurement of the perception threshold of heat and cold sensations has been reviewed in a previous paper. ${ }^{2}$ We have shown that by modification of some of the available methods, reliable quantitative and reproducible indices of the

Address for reprint requests: Dr Goran A Jamal. Department of Neurology, Institute of Neurological Sciences, Southern General Hospital, Glasgow G51 4TF, Scotland, UK

Received 15 June 1984 and in revised form 22 August 1984. Accepted 23 August 1984. functional integrity of these endorgans and fibre populations, can be obtained. ${ }^{2}$ We believe that this method may be useful for the detection of dysfunction in the small fibre population of peripheral nerves as an early manifestation of peripheral neuropathy and to detect small fibre damage in ongoing generalised neuropathy.

The present paper describes the result of application of this new technique for thermal threshold measurement in a large group of patients with neuropathy from different causes. The primary aim was threefold: (1) to illustrate the clinical usefulness of the technique, (2) to evaluate the frequency of involvement of the small unmyelinated and thinly myelinated fibres in neuropathy in general, (3) to compare the sensitivity of this technique with electromyography and nerve conduction studies in the early diagnosis and the ongoing assessment of peripheral neuropathy. 


\section{Methods}

Electromyography and nerve conduction (EMG and NC) studies

These were undertaken on a Medelec MS6 electromyograph. The fastest motor conduction velocity (FMCV) and the shortest distal motor latency (SDML) for the right (R) common peroneal and median nerves were obtained recording from surface electrodes over the target muscle by conventional techniques. ${ }^{3}$ The amplitude and duration of the compound muscle action potential, so evoked, were also measured. Sensory action potentials (SAP) were elicited orthodromically in the $\mathbf{R}$ median, ulnar and sural nerves. For each SAP measurement, 64 evoked potentials were averaged. Sensory latencies were measured from the onset of the stimulus artefact to the peak of negative deflection. Amplitudes were measured from peak to peak. For median and ulnar nerve sensory potentials, the method used by Gilliatt and Sears ${ }^{4}$ was followed. The sural nerve was stimulated at the lateral aspect of the foot immediately inferior and anterior to the lateral malleolus and the potential recorded by surface electrodes $12-14 \mathrm{~cm}$ proximal to the malleoli lateral to the tendo Achillis. The distance between the recording and the reference electrodes was $4 \mathrm{~cm}$. Electromyographic studies were performed with concentric bipolar needle electrodes in one or more of the following muscles: the $\mathbf{R}$ extensor digitorum brevis, the $\mathbf{R}$ tibialis anterior and the first dorsal interosseous muscles. The ambient room temperature was kept at $22 \pm 2^{\circ} \mathrm{C}$. Skin temperature of the limb was maintained at $34 \pm 1^{\circ} \mathrm{C}$ using a thermostatically controlled heating lamp.

\section{Thermal thresholds measurement}

Heat thresholds (HT) and cold thresholds (CT) were determined using a microcomputer controlled automated system incorporating the two alternative forced-choice method of psychophysical analysis. ${ }^{2}$ Two distal sites, relevant for examination of polyneuropathy, were selected.
These were the volar aspect of the $R$ wrist just proximal to the distal crease and the medial aspect of the $R$ ankle behind the medial malleolus, two sites identical to those examined in the control group. The ambient temperature of the room was kept constant at $22 \pm 2^{\circ} \mathrm{C}$. Thermal threshold determinations for controls ${ }^{2}$ and patients were carried out by the same person (GAJ).

\section{Patients and control subjects}

The control subjects for the thermal thresholds consisted of 106 healthy volunteers aged between $6-73$ (mean $=33$; $\mathrm{SD}=17$ ) years. Full description of selection of these subjects and their HT and CT values are described separately. ${ }^{2}$ The control group for the EMG and NC studies were 42 healthy subjects aged between $18-59$ (mean $=35 ; \mathrm{SD}=$ $10)$ years. There were 143 patients with polyneuropathy of varied severity and aetiology. Most were ambulant and in ? good general health. All were cooperative and informed. Their age ranged between 9-77 (mean $=49 ; S D=16)$ years. The mean duration of neuropathy was 48 months with a range of 2 days -40 years. The causes of the neuropathy are summarised in table 1 .

Full general and neurological examination was performed on all the patients and any abnormalities of touch, pinprick and vibration were noted. Full laboratory examination was undertaken to determine the aetiology of the neuropathy. ${ }^{56}$ The diagnosis in the suspected cases of hereditary aetiology was established by the clinical examp nation and laboratory investigation of the patients and relatives.

The aetiology of the polyneuropathy was determined $70 \%$ of the patients. In $47 \%$ an acquired cause (toxi metabolic-endocrine, autoimmune, paramalignant of or allergic) was responsible whle hereditary factors were operative in $23 \%$. In the remaining $30 \%$, no cause was discovered (table 1).

Table 1 Classification of the 143 neuropathy cases by aetiology

\begin{tabular}{|c|c|}
\hline Aetiology & Number and percentage \\
\hline $\begin{array}{l}\text { Acquired known cause (total) } \\
\text { Diabetes mellitus } \\
\text { Hypothyroidism } \\
\text { Alcoholic neuropathy } \\
\text { Blood disorders (folate, Vit B12 deficiency) } \\
\text { Rheumatoid arthritis } \\
\text { Porphyric neuropathy } \\
\text { Carcinoma associated } \\
\text { Guillaine Barré Syndrome } \\
\text { Heavy metals (for example, mercury, lead) } \\
\text { Drugs (for example, phenytoin, aminodarone, thalidomide, isoniazid) }\end{array}$ & $\begin{array}{l}67(46 \cdot 8 \%) \\
30 \\
3 \\
10 \\
3 \\
2 \\
1 \\
2 \\
4 \\
5 \\
7\end{array}$ \\
\hline $\begin{array}{l}\text { Hereditary neuropathies (total) } \\
\text { HMSN type I } \\
\text { HMSN type II } \\
\text { HMSN type IV (Refsum's syndrome) } \\
\text { HSN type II } \\
\text { Friedreich's ataxia } \\
\text { Hereditary spastic paraplegia and neuropathy } \\
\text { Retinitis pigmentosa and neuropathy } \\
\text { Idiopathic (total) }\end{array}$ & $\begin{array}{l}33(23 \cdot 1 \%) \\
13 \\
9 \\
1 \\
1 \\
5 \\
3 \\
3 \\
43(30 \cdot 1 \%)\end{array}$ \\
\hline
\end{tabular}

HMSN = hereditary motor sensory neuropathy

$\mathrm{HSN}=$ hereditary sensory neuropathy 
Table 2 Clinical, conventional electrophysiological and thermal threshold assessment of 143 patients with neuropathy

\begin{tabular}{lc}
\hline Evidence for neuropathy & Number and percentage \\
\hline One or more symptoms of neuropathy (total) & $109(76 \%)$ \\
pain & 53 \\
paraesthesia & 86 \\
numbness & 63 \\
weakness and/or fasciculation & 81 \\
autonomic & 42 \\
altered thermal feeling & 69 \\
One or more signs of neuropathy (total) & $114(79 \cdot 7 \%)$ \\
abnormal touch & 77 \\
abnormal vibration & 76 \\
abnormal pinprick & 94 \\
weakness, wasting and/or fasciculation & 80 \\
Abnormal EMG and/or NC studies (total) & $127(88 \cdot 8 \%)$ \\
abnormal one or more SAP & 103 \\
abnormal sural SAP & 95 \\
abnormal ulnar and/or median SAP & 73 \\
Abnormal one or more thermal threshold (total) & $141(98 \cdot 6 \%)$ \\
Abnormal ankle: HT CT & 125 \\
HT \& CT & 127 \\
Abnormal wrist: HT CT HT & 114 \\
HT \& CT & 141
\end{tabular}

EMG \& NC: electromyography and nerve conduction

SAP: sensory action potential

HT: heat threshold

CT: cold threshold

\section{Results}

Table 2 summarises the clinical, electrophysiological and thermal studies on these patients. Abnormality of thermal thresholds was considered to be present if values exceeded the 99 th percentile. Abnormality of conduction was considered to be present if values exceeded the 95 percentile of the corresponding normal mean. Normal values for the thermal thresholds are described in detail elsewhere. ${ }^{2}$ Normal values for nerve conductions are summarised in Table 3. One hundred and nine of the 143 patients studied $(76 \%)$ had one or more symptoms known to be associated with neuropathy ${ }^{7}$ while $114(79.7 \%)$ showed some abnormality of sensation on clinical examination for - one or more of the modalities tested. EMG and/or NC studies were abnormal in $127(88.8 \%)$ patients, of whom $103(72 \%)$ had an abnormality. of the amplitude, duration and/or latency of one or more of the SAPs determined. In 40 patients $(28 \%)$ all of these parameters (amplitude, duration and latency) of the ulnar, median and sural SAPs were within the normal range. Sixty-eight of the $143(47 \cdot 5 \%)$ showed abnor-

Table 3 Normal nerve conduction values for 42 healthy subjects at skin temperature of $34 \pm 1^{\circ} \mathrm{C}$

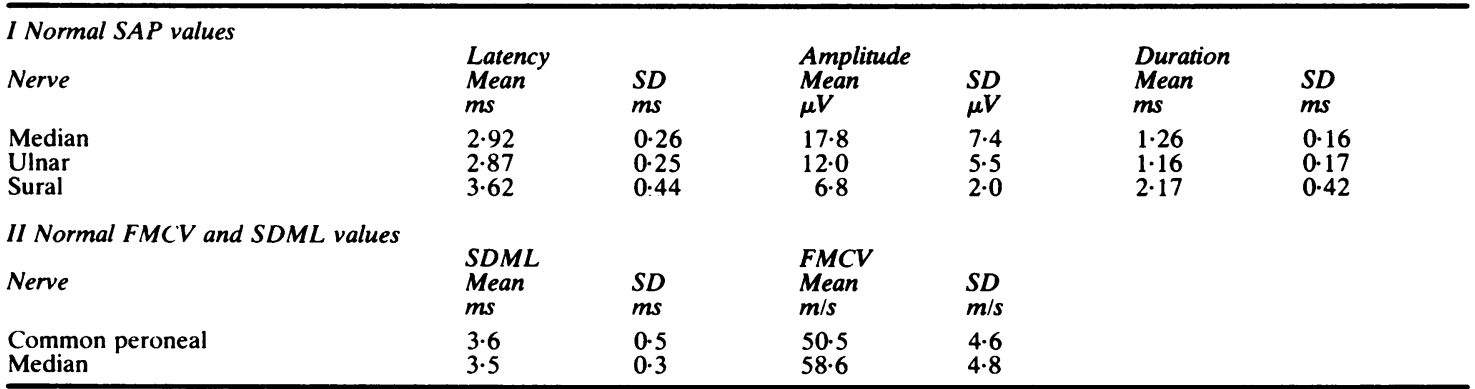

SAP: sensory action potential (orthodromic, surface recording)

FMCV: fastest motor conduction velocity

SDML: shortest distal motor latency

SD: standard deviation 

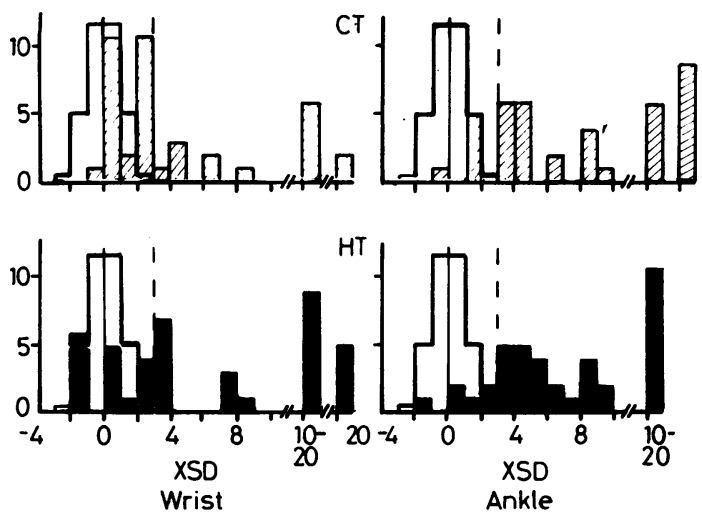

Fig 1 Ankle and wrist thermal thresholds in 40 patients with normal sensory median, ulnar and sural nerve studies on conventional electrophysiology; vertical axis = number of patients; horizontal axis = multiples of standard deviation $(\times S D)$ from normal control mean; open histogram $=$ normal control values distribution; solid vertical line $=$ mean value for normal controls; dashed vertical line $=3 \times S D$ above normal control mean; $H T=$ heat threshold; $C T=$ cold threshold.

malities in the ulnar and/or median SAP. Ninety-five of the $143(66 \%)$ showed abnormalities in the sural nerve SAP. One or more of the thermal thresholds determined were abnormal (outside the 99th percentile) in 141 patients $(98.6 \%)$. Only one patient, a recently diagnosed case of Friedreich's ataxia, with abnormal sural SAP had normal thermal thresholds.
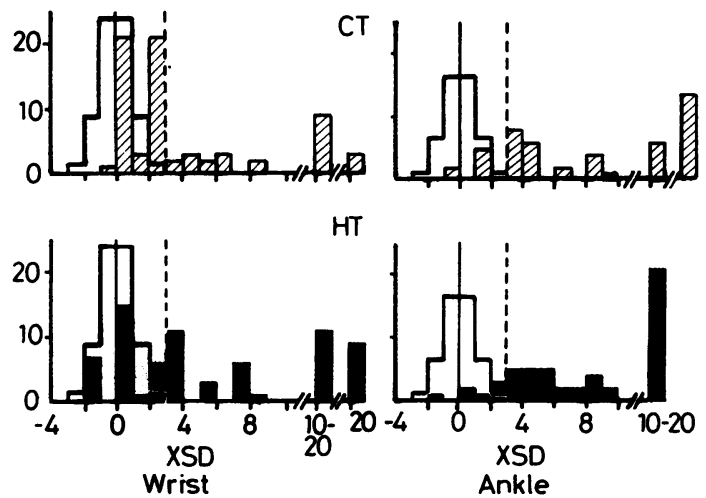

Fig. 2

Fig. 3

Fig 2 Wrist thermal thresholds in 70 patients with normal sensory ulnar and median nerve studies on conventional electrophysiology. (symbols as in fig 1)

Fig 3 Ankle thermal thresholds in 48 patients with normal sensory sural potential on conventional electrophysiology. (symbols as in fig 1)
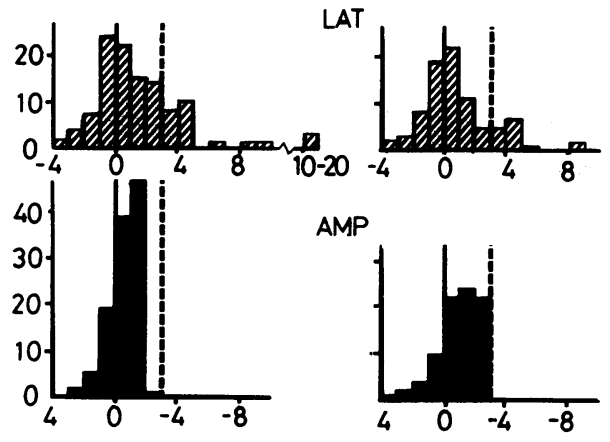

AMP

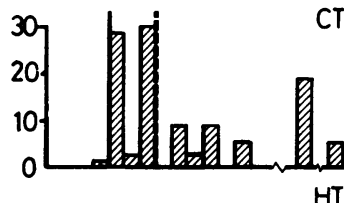

T
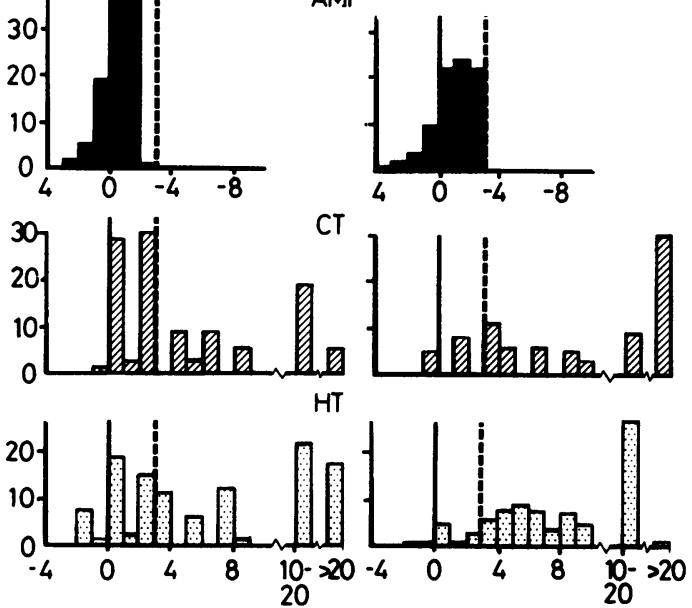

Median nerve -wrist ( $n=116$ ) Sural nerve-ankle ( $n=85)$

Fig 4 Comparison of conventional sensory electrophysiological studies and thermal thresholds in patients with neuropathy $\dagger$. vertical axis = number of patients; horizontal axis = multiples of standard deviation $(\times S D)$ outwith the normal control mean*; solid vertical lines = mean value for normal controls; dashed vertical lines $=3 \times S D$ above normal control mean; $L A T=$ latency of the sensory action potential (SAP); $A M P=$ amplitude of the SAP; $C T=$ cold threshold; $H T=$ heat threshold; tpatients with absent median SAP (when compared with wrist thermal thresholds) and those with absent sural SAP (when compared with ankle thermal thresholds) are excluded. "negative values are on the right side of zero in the AMP histogram as these represent abnormalities of amplitude.

The other patient, a female aged 17 years also with a recently diagnosed Friedreich's ataxia, had both the thermal threshold values and the sural SAP studies within normal limits. Abnormalities of thermal thresholds were generally more marked at the ankle $(98.6 \%)$ than the wrist $(71.3 \%)$. It is noteworthy that in nine asymptomatic patients with minor abnormalities in EMG, known to have disease predisposing to neuropathy, there was no abnormality on NC studies. These nine patients were found to have significant abnormalities of one or more of the thermal thresholds.

\section{Discussion}

The results of the application of the Glasgow $\underset{<}{ }$ 

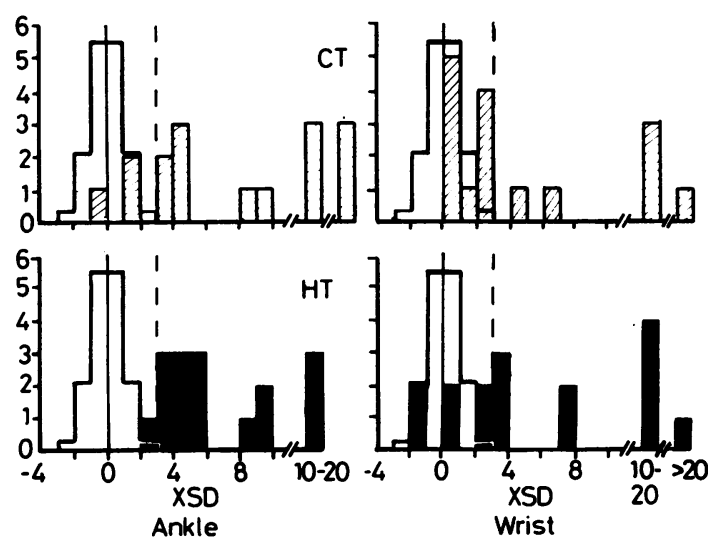

Fig 5 Ankle and wrist thermu thresholds in 16 patients with clinical evidence of neuropathy but normal conventional electrophysiological (EMG and NC) studies. (symbols as in fig 1)

method to patients with peripheral neuropathy underlines its usefulness in these disorders. Abnormalities of thermal sensation were detected in 141 patients $(98.6 \%)$ compared to $79.7 \%$ on clinical examination of the sensory system and $72 \%$ on sensory conduction studies (table 2 ). While significant alterations of thermal thresholds were most often observed in patients with concomitant abnormalities on clinical and electrical test, they also occurred in isolation. Similar observations have been reported by other workers using different methods. ${ }^{8}$ Among 40 patients with normal SAP studies in the median, ulnar and sural nerves (fig 1), 34 (85\%) had ankle HT and CT outside the 99th percentile while 20 (50\%) had abnormal wrist HT and CT values. One of more thermal thresholds were abnormal in 39 $(97.5 \%)$ of the 40 patients. Figure 2, from 70 patients with normal ulnar and median SAPs, shows wrist HT and CT values expressed in multiples of the standard deviation $(\times S D)$ for the control group outside the normal mean. In $42(60 \%)$ of these, one or both wrist thermal threshold values were abnormal. Figure 3 , from 48 patients who had normal sural SAP, shows ankle HT and CT values. These thermal threshold values were abnormal in $86 \%$ of the patients.

Although abnormality within the central nervous system cannot be entirely excluded in patients with neuropathy, with certain exceptions (for example, hereditary neuropathies) one can assume that the abnormal thermal threshold values were mostly due to dysfunction of the peripheral fibre population subserving thermal sensation and/or their end organs. The observation that fewer patients had abnormal values from wrist compared to ankle, is compatible with the increasing severity of dysfunction distally in peripheral neuropathy.

The SAP parameters, indices of the functional integrity of the large diameter afferent fibres, and the thermal thresholds, indices of the functional integrity of the small diameter afferent fibres, were compared. Patients with absent SAP, all of whom had abnormal thermal threshold values, were excluded. Latencies and amplitudes of the $\mathbf{R}$ median and sural nerves were compared with HT and CT of the wrist and the ankle respectively. To facilitate this comparison, each value was expressed as a figure representing the $\times S D$ outside the corresponding normal mean and the histograms plotted in fig 4 . Again the standard deviation used is that of the corresponding control parameter. None of the SAP amplitude values was more than $3 \times$ SD outside the normal mean and few had their SAP latency values outside this value. The majority of patients had their thermal threshold values in excess of $3 \times \mathrm{SD}$ above the normal mean. This emphasises the sensitivity of our technique in assessing small fibre function compared to available conventional methods.

The thermal thresholds for wrist and ankle were also correlated with the corresponding SAP parameters. Patients with absent SAP were again excluded. The most consistently significant correlation was with the SAP amplitude, a measure of loss of large afferent fibres in neuropathy.9 $A$ reduction in the median (or ulnar) SAP amplitude was accompanied by an elevation of the wrist HT $(p<0.05)$ and CT $(p<0.05)$. The same correlation was obtained between the sural SAP amplitude and ankle HT $(p<0.02)$ and CT $(p<0.001)$ values. These findings suggest that in most neuropathies a more or less parallel drop occurs in both fibre population groups but not necessarily to the same extent in each type of neuropathy. This appears to be true of the neuropathies in general. ${ }^{10} 11$

In 16 patients, no abnormality of EMG and NC studies was found. All, except one with recently diagnosed Friedreich's ataxia, had symptoms and signs of peripheral nerve dysfunction. Another patient with paraesthesiae in the feet, had taken phenytoin for several years. The remaining 14 of the 16 had clinical features to suggest involvement of small fibres, for example spontaneous pain in the extremities, diminished pain and temperature sensation and autonomic features, for example orthostatic hypotension, disturbances of sweating, disturbances of genito-urinary function (intermitted stream, post micturition dribbling, retention with overflow incontinence and failure of erection and ejaculation), disturbances of bowel function and nocturnal diarrhoea. ${ }^{1112}$ Muscle strength, tendon reflexes and other sensory modalities (touch- 
pressure, vibration and joint position) were preserved in these patients. Diabetes mellitus was the underlying cause in eight but no obvious cause was discovered in the remaining six patients. Figure 5 gives a summary of the thermal threshold values for these 16 patients. All the 14 patients with small fibre dysfunction and the patient on phenytoin had abnormal thermal thresholds. In the patient with Friedreich's ataxia, all the threshold values were normal. Normal thermal threshold values have been observed before in patients with Friedreich's ataxia, in which the large afferent fibres are more severely affected. ${ }^{12}$ This technique, therefore, is specifically useful in cases of neuropathy whiere small fibre dysfunction may be the earliest or the only abnormality and also in assessing a concomitant small fibre dysfunction in somatic neuropathies.

In conclusion, the data presented in this study clearly show the superiority of our technique over clinical testing in detecting small fibre involvement in peripheral neuropathy even in the absence of clinical or other electrophysiological evidence of dysfunction. In combination with other electrophysiological techniques testing large afferent fibre function, it gives a good idea about the pattern of involvement of various fibre populations in peripheral neuropathies.

We are grateful to Professor J A Simpson for support and advice. We also thank all our colleagues in the Department of Neurology for referring patients and Mrs M McColl for secretarial assistance. Financial support was received from The Department of Neurology Research Fund, University of Glasgow. Dr G A Jamal was supported by a grant from Baghdad University, Iraq.

\section{References}

' Light AR, Perl ER. Peripheral sensory systems. In Dyck PJ, Thomas PK, Lambert EH and Bunge $R$ eds.
Peripheral Neuropathy Londón: WB Saunders $1984 ;$ Z $_{\mathbb{D}}$ Vol 1 19:210-30.

2 Jamal GA, Hansen S, Weir AI, Ballantyne JP. An' improved automated method for the measurement of thermal thresholds. 1. Normal subjects. J Neurol Neurosurg Psychiatry (in press).

${ }^{3}$ Lenman JAR, Ritchie AE. Clinical Electromyography 2nd ed. Bath: Pitman 1977;60-72.

4 Gilliatt RW, Sears TA. Sensory action nerve potentials in patients with peripheral nerve lesions. J Neurol. . Neurosurg Psychiatry 1958;21:109-18.

5 Dyck PJ, Oviatt KF, Lambert EH. Intensive evaluation of referred undiagnosed neuropathies yields improved $\bar{C}$ diagnosis. Ann Neurol 1981;10:222-6.

${ }^{6}$ McLeod JG, Polard JD. Neuropathies in systemic dis- $\frac{\bar{C}}{}$ eases: hidden and overt. In: Asbury AK and Gilliatt $\mathbb{D}$ RW eds. Peripheral Nerve Disorders. London:
Butterworths. 1984;92-125.

7 Thomas PK. Symptomatology and differential diagnosis $\overrightarrow{0}$ of peripheral neuropathy; clinical features and differ-ential diagnosis. In: Dyck PJ, Thomas PK, Lambert $\vec{\omega}$ EH, Bunge R eds. Peripheral Neuropathy. London: WB Saunders. 1984; Vol 1:1169-1190.

${ }^{8}$ Fruhstorfer H, Goldberg JM, Lindblom U, Schmidt WG? Temperature sensitivity and pain thresholds in ${ }_{\infty}^{+}$ patients with peripheral neuropathy. In: Zotterman $Y_{\perp}$ ed. Sensory Functions of the Skin in Primates. Oxford: $\omega$ Pergamon Press 1976;507-517.

${ }^{9}$ Buchthal F, Rosenfalck A, Behse F. Sensory potentialso of normal and diseased nerves. In: Dyck PJ, Thomas PK, Lambert EH and Bunge $R$ eds. Peripheral ${ }_{D}$ Neuropathy. London: WB Saunders. 1984; BI을 1:981-1015.

${ }^{10}$ Adams RD, Victor M. Other disorders of somatic sen tion. In: Principles of Neurology. New York: McGrajw Hill. 1981; 2nd ed, :103-116.

$"$ Ochoa J. Recognition of unmyelinated fibre disease:O morphologic criteria. Muscle Nerve 1978;1:375-87.

12 Dyck PJ, Lambert EH, Nichols' PC. Quantitative meas- $\bar{O}$ urement of sensation related to compound action potential and number and sizes of myelinated and unmyelinated fibers of sural nerve in health, Friedreich's ataxia, hereditary sensory neuropathy and tabes dorsalis. In Cobb WA ed. Handbook of Elec- troencephalography and Clinical Neurophysiology Amsterdam: Elsevier. 1972; Vol 9:83-118. 